\title{
Comunidades virtuales de viajeros: un caso de éxito
}

\section{Travellers' virtual communities: a success story}

\author{
Laura Cervi \\ Universidad Autónoma de Barcelona (España) \\ Departamento de Periodismo y Ciencias de la Comunicación \\ laura.cervi@uab.cat \\ Código Orcid: www.orcid.org/0000-0002-0376-0609
}

\section{Resumen}

Este artículo se propone estudiar la comunidad italiana de viajeros on line Ho sempre voglia di partire que, en solo dos años de vida, cuenta con más de 540000 seguidores, más de 7000000 de visitas por mes y 2 700000 interacciones.

El objetivo es describir las interacciones presentes en la comunidad para desvelar las razones por las cuales los usuarios participan, los beneficios, reales y simbólicos que obtienen, y poder comprender las razones del éxito. A través de una triangulación de métodos el artículo desvela como la clave de todas las interacciones es la emoción y que, por lo tanto, los beneficios que los miembros obtienen son principalmente emocionales.

\section{Palabras clave}

Redes sociales, comunidades virtuales, comunidades de viajeros, viajeros 2.0 , Web 2.0 , community manager, prosumidor, turismo 2.0 .

\begin{abstract}
This article aims at studying the Italian online travelers' community Ho sempre voglia di partire, which, in only two years of life, has reached more than 540000 followers, over 7000000 visits per month and over all 2700000 interactions, becoming - according to its creators - the largest travelers' community in Europe. The objective is to describe the interactions that characterizes the community, to reveal the reasons why users participate, the actual and symbolical benefits they obtain, and to understand the reasons for success. Through a triangulation of methods, the article reveals how the key of all interactions is emotion and how, therefore, the benefit that the members obtain is mainly emotional.
\end{abstract}

\section{Keywords}

Social networks, on line communities, travel communities, 2.0 travellers, Web 2.0, community manager, prosumer, 2.0 tourism.

Forma sugerida de citar: Cervi, Laura (2019). Comunidades virtuales de viajeros: un caso de éxito. Universitas, 30, pp. 97-125. 


\section{Introducción}

El viaje siempre ha sido una necesidad del individuo: primero con los griegos que viajaron por el deseo de saber, ver y aprender, luego con los romanos que comenzaron a practicar el "viaje de placer" hasta que llegamos a nuestros días, donde las razones que nos motivan a viajar son innumerables.

El uso de internet y las tecnologías digitales ha revolucionado radicalmente la forma de viajar: en lugar de acudir las agencias de viajes, usamos sus sitios en línea, o directamente prescindimos de ellas al planificar y organizar nuestros propios viajes personalizados con vuelos, reservas de hoteles y servicios en línea.

El turismo, de hecho, se ha clasificado como la industria más importante en términos de volumen de transacciones en línea (Werthner \& Ricci 2004). Para las organizaciones turísticas, tanto privadas como públicas, por lo tanto, internet se ha convertido en uno de los canales de comunicación y de marketing más importantes (Wang \& Fesenmaier 2006).

Pero internet ha revolucionado sobre todo el modelo de búsqueda de información.

El modelo de comunicación vertical y unidireccional de la sociedad industrial y de los medios de comunicación de masa, de hecho, ha sido reemplazado por uno horizontal y multidireccional, en el que los individuos son tanto productores como consumidores de contenido, tanto emisores como receptores.

Es lo que Axel Bruns (2008) denominó como "produsage", haciendo referencia a la naturaleza dual del ciudadano digital que se convierte en prosumer ya que consume y produce a la vez información como un actor comunicativo más.

Lo mismo ha pasado en el mundo del viaje: las tecnologías de los medios sociales han llevado a un cambio en el control de los procesos de creación de contenidos, desde una Web 1.0 principalmente controlada por organizaciones y corporaciones (Li \& Bernoff, 2008; Qualman, 2009) hacia el enfoque más inclusivo de la Web 2.0, que en gran medida es expresión de interacción y participación del usuario final (Kamboj \& Rahman, 2017; Rashidi et al., 2017).

Hoy día cualquier usuario puede acceder a la web para descubrir y discutir las experiencias de otras personas: estas "comunidades", sitios que conectan a personas en todo el mundo están, en efecto, eclipsando incluso a los tradicionales portales y motores de búsqueda (Prebensen, Kim \& Uysal, 2016). 
Este nuevo patrón de uso fue definido por Philipe Wolf, Director General de PhoCusWright Inc., en 2006, como "viaje 2.0", es decir en el uso de herramientas de la web 2.0 como blogs, redes sociales, sistemas de recomendación, integración de contenidos mediante mashups, audio, video, planificadores de viaje, etc. Sin embargo, en la actualidad el concepto abarca mucho más, ya que no supone tan sólo el uso de las herramientas para buscar información sino, fundamentalmente, un cambio de paradigma, en la filosofía global del uso de la información: el usuario utiliza las tecnologías en el proceso de anticipación, experiencia y recreación del viaje (Almeida et al., 2016).

Las revistas de disciplinas económicas, especialmente el marketing (Sotiriadis, 2017; Roque \& Raposo 2016), y las de turismo (véase, entre otros, Amaro, Duarte \& Henriques, 2016; Kavoura \& Borges, 2016), han dedicado muchos estudios a las comunidades de viajeros, con un enfoque claramente business-oriented, sin embargo las ciencias de la comunicación parecen no haberse interesado mucho a estas comunidades.

Por esta razón el artículo se propone estudiar un caso particularmente exitoso, la comunidad italiana de viajeros on line Ho sempre voglia di partire (literalmente "siempre tengo ganas de salir", pero el verbo partire en italiano se acerca más a "despegar", por esto la traducción más adecuada nos parece "Tengo siempre ganas de viajar"), que en solo dos años de vida cuenta, según los datos oficiales, con más de 540000 seguidores, más de 7000 000 de visitas por mes y sobre todo 2700000 interacciones, convirtiéndose, según sus creadores, en la comunidad de viajeros más grande de Europa. El objetivo es describir las interacciones presentes en la comunidad para desvelar las razones por las cuales los usuarios participan, los beneficios, reales y simbólicos que obtienen, y poder comprender las razones del éxito en términos de interacción entre usuarios.

\section{Estado de la cuestión}

Cuando hablamos de viaje y de internet la mayor parte de los estudios pertenecen a las áreas del marketing, o de turismo.

Desde hace décadas estas disciplinas han descrito cómo los consumidores buscan información (Howard, Restrepo \& Chang, 2017). 
Los productos de viaje son intangibles y no se pueden evaluar por adelantado, por lo tanto, cuando los consumidores planifican viajar tienden a realizar una extensa búsqueda de información para reducir el riesgo y la incertidumbre (Gitelson \& Crompton, 1983; Mansfeld, 1992; Mayo \& Jarvis, 1981; Mill \& Morrison, 2002; Filieri \& McLeay, 2014; Chang, Fu \& Jain, 2016). Además, viajar y tomar vacaciones es una de las partidas más grandes en el presupuesto anual (Mill \& Morrison, 2002; Sirakaya \& Woodside, 2005), de modo que, los posibles viajeros a menudo intentan maximizar el conocimiento buscando tanto como sea posible la información (Yang \& Bin Guo, 2016).

El patrón de búsqueda de información, además, a menudo se ve influenciado por los perfiles demográficos, los niveles de experiencias y un rango de otras variables (Andereck \& Caldwell, 1993; Wang, et. Alt.2010; Shneiderman, 2015).

\section{User generated content y viaje}

Como ya hemos anticipado, la aparición de las TIC ha cambiado la forma de comprar productos y servicios relacionados con los viajes, debido a su funcionalidad de comunicación interactiva y bidireccional (Buhalis, 2003).

En particular la Web 2.0, crea nuevos tipos de fuentes de información: los viajeros potenciales están expuestos a muchos tipos diferentes de información a partir de una gran cantidad de proveedores.

Al lado de las tradicionales fuentes de información - principalmente medios, instituciones y empresas-, de hecho los consumidores generan cada vez más su propio contenido a través de cámaras digitales, cámaras web, teléfonos con imágenes, comunidades en línea y blogs web (Chen, Yang \& Tang , 2013; Gretzel, Fesenmaier \& O’Leary, 2006). Los miembros de la comunidad en línea pueden publicar sus propias experiencias, compartir su opinión, dar consejos o buscar respuestas a sus preguntas (Mohd-Any, Winklhofer \& Ennew; 2015; Olsen \& Connolly, 2000).

En otras palabras así como en la Web 2.0 todos los usuarios pueden participar activamente en la generación o enriquecimiento de contenido (Kaplan \& Haenlein, 2010), del mismo modo, los viajeros de hoy pueden participar activamente en el consumo, la producción y la difusión de información de viajes a través de Internet (Pantelidis, 2010; Sparks \& Browning, 2011). 
El fenómeno de los turistas que crean y comparten información en línea ha recibido mucha atención a la investigación. La mayoría de los primeros estudios, de enfoque exploratorio y descriptivo, se han centrado en las mismas plataformas (Enoch \& Grossman, 2010; Litvin, Goldsmith, \& Pan, 2008; Pudliner, 2007; Schmallegger \& Carson, 2008; Wenger, 2008), o en temas específicos, como por ejemplo las principales vacaciones de verano (Bronner \& de Hoog, 2011), evidenciando cómo las redes sociales están ganando en cuanto a prácticas de comunicación y de viaje emergentes y por otra parte, también discuten críticamente la calidad de las contribuciones de los viajeros y limitaciones de las prácticas comunicativas en línea (Jacobsen \& Munar, 2012; Volo, 2010).

Además, algunos estudios han analizado los factores de motivación que afectan el comportamiento en línea de los viajeros (Bryce, Curran, O' Gorman \& Taheri, 2015; Chung, Lee \& Koo, 2015). Según Hsu, Ju, Yen y Chang (2007), la disposición a compartir el conocimiento en línea depende de la cognición personal y la influencia social.

En particular, el intercambio de información sobre viajes a través de blogs ha recibido una amplia atención de parte de investigadores en turismo (Enoch \& Grossman, 2010; Huang, Chou \& Lin, 2010; Mack, Blose \& Pan, 2008; Schmallegger \& Carson, 2008; Tussyadiah \& Fesenmaier, 2008; Volo, 2010; Wenger, 2008).

Los estudios más recientes (Ert, Fleischer \& Magen, 2016; Harrigan, Evers, Miles \& Daly; 2017) sugieren que las revisiones de viajes en línea a menudo se perciben como más genuinas y aptas a proporcionar información confiable que el contenido publicado por las organizaciones de turismo. Por esto cada vez más las comunidades en línea son consideradas fuentes de información más influyentes (Mohd-Any, Winklhofer \& Ennew, 2015), y si la membresía genera contenido de buena calidad y se mantiene enérgicamente, a menudo se perciben como una recomendación similar a la de amigos, familiares, es decir como sustitutos del boca a boca (Dedeke, 2016; Bray, Schetzina \& Steinbrink, 2006).

Algunas comunidades como VirtualTourist.com, Trekshare.com, Lonelyplanet.com, y Tripadvisor.com ya desempeñan un papel fundamental en el suministro de información actualizada sobre destinos de miembros de todo el mundo (Litvin \& Dowling, 2017; Kavoura \& Borges, 2016; Beith, 2004). 


\section{Viajes y comunidades on line}

Una comunidad en línea puede verse como un ágora virtual y un mercado en el que la información se comparte y los consumidores generan su contenido (Wang et al., 2002).

Muchos investigadores, en diferentes disciplinas, han intentado describir las comunidades en línea y captar sus características (Preece, 2000; Armstrong \& Hagel, 1996; Powers, 1997; Rheingold, 1991; Rosenblatt, 1997; Shelton \& McNeeley, 1997; Smith \& Kollock, 1999).

En particular, los sociólogos han intentado durante años definir el concepto y las características de una comunidad (Prebensen, Kim \& Uysal, 2016; Reypens, Lievens \& Blazevic, 2016), sin embargo, la investigación sobre comunidades virtuales en línea todavía está en su infancia en comparación con la investigación sobre comunidades definidas geográficamente y físicas (Preece, 2000).

La clave para comprender una comunidad es comprender las motivaciones y las necesidades que subyacen detrás de la participación de cada miembro (Ayeh, 2015; Kim, Lee \& Hiemstra, 2004; Kozinets, 1999): por eso muchos investigadores han estado tratando de identificar los beneficios percibidos de los miembros en comunidades virtuales (Bilgihan, Barreda et al., 2016; Ayeh, Au \& Law, 2013b).

En el mundo del viaje, si bien las personas obviamente buscan información para la toma de decisiones (Amaro \& Duarte, 2013; Agag \& El-Masry, 2016b), también es evidente que las personas que recopilan información sobre viajes no necesariamente tienen una intención real de viajar (Chung \& Koo, 2015; Urry, 1990; Woodside, 1990) sino que a menudo están simplemente interesados en conocer a personas de ideas afines, con actitudes, intereses o estilos de vida similares (Agag \& El-Masry, 2016a; Kang \& Schuett, 2013; Wang, Yu \& Fesenmaier, 2002).

Es decir, más allá de las necesidades funcionales, como la búsqueda de información necesaria para realizar un viaje en concreto, las personas usan la información como una ocasión para compartir con otros, o simplemente disfrutar (Kavoura \& Stavrianea, 2015). Armstrong y Hagel (1997) demuestran que una comunidad en línea proporciona cuatro valores diferentes a los miembros: transacción, interés, fantasía y relación. Vogt y Fesenmaier (1998) argumentan que las necesidades de información se expanden más 
allá de las necesidades funcionales, hacía cuatro dimensiones adicionales: necesidades hedónicas, de innovación, estéticas y de signos.

En línea con los hallazgos de investigaciones anteriores, Wang y Fesenmaier (2004b) argumentan que los beneficios sociales y hedónicos tienen un mayor impacto en la participación de los miembros en las actividades de la comunidad en línea que los beneficios funcionales. Los beneficios sociales se refieren a la comunicación con otros miembros, la construcción de relaciones, el intercambio de ideas y opiniones y la participación (Angehrn, 1997; Preece, 2000; Wang \& Fesenmaier, 2004a).

Con base en 346 usuarios de Internet de Rusia y de las antiguas repúblicas de la Unión Soviética, Fotis et al. (2012), por ejemplo, evidenciaron que las redes sociales se utilizan principalmente después de las vacaciones para compartir experiencias.

En conclusión, para comprender el fenómeno de las comunidades de viajes en el contexto en línea es importante examinar lo que realmente motiva a los usuarios en línea a involucrarse en estas comunidades con actitudes positivas.

Baym (2010) ha propuesto un marco conceptual basado en siete dimensiones para categorizar las comunidades: interactividad, que indica diferentes niveles de interactividad social, habilitada por diferentes plataformas comunicativas; estructura temporal, que reflexiona sobre prácticas asíncronas y síncronas y características en tiempo real; señales sociales, que examinan la riqueza del contexto (por ejemplo, información sobre identidades personales y contextos espaciales y ambientales); alcance, que mide la audiencia que un medio puede alcanzar o apoyar; movilidad, que se refiere a la medida en que los medios son portátiles, lo que permite a las personas comunicarse casi sin importar la ubicación; almacenamiento y replicabilidad, refiriéndose a las posibilidades de grabación y reproducción.

\section{Método}

Para estudiar la comunidad Ho sempre voglia di partire hemos aplicado un método mixto (Creswell, 2014, p. 2), basado en la triangulación de cuatro métodos: netnografía, web-analysis (Rieder, 2013), análisis del contenido y entrevista en profundidad con su creador y creador de todos los contenidos, Guido Prussia. 
La palabra netnografía, combinación de "Internet" o "red" con "etnografía", fue creada originalmente en 1995 por Robert Kozinets como una herramienta para analizar las discusiones de los fanáticos en línea sobre la franquicia de Star Trek. El uso del método se extendió desde la investigación de mercado y la investigación del consumidor a una variedad de otras disciplinas, convirtiendo la netnografía es una disciplina aún en construcción y heredera de disciplinas clásicas como la antropología social y cultural, la sociología e incluso de la psicología con el objetivo de entender la realidad social que se está produciendo en el contexto online donde millones de personas conviven, se expresan e interactúan a diario.

Se define como un conjunto específico de prácticas de investigación relacionadas con la recopilación de datos, el análisis, la ética de la investigación y la representación, enraizadas en la observación participante. En netnografía, una cantidad significativa de los datos se origina y se manifiesta a través de las huellas digitales de las conversaciones públicas que ocurren naturalmente, registradas por las redes y utiliza estas conversaciones como datos. Por esta razón, ofrece una experiencia de investigación menos intrusiva que la etnografía, ya que utiliza principalmente datos de observación. En comparación con la etnografía tradicional, que requiere que los investigadores se sumerjan físicamente en las muestras para recopilar datos, los investigadores netnográficos pueden descargar datos de comunicación directamente desde una comunidad en línea, permitiendo al investigador investigar un gran número de personas.

Con la influencia de la etnografía, este método de investigación permite al investigador vincular los patrones de comunicación para comprender las prácticas tácitas y latentes involucradas dentro y entre estas comunidades de interés en línea (Mariampolski, 2005). Como señaló Kozinets (1999, p. 366), "estos grupos sociales tienen una existencia" real "para sus participantes, y por lo tanto tienen efectos consecuentes en muchos aspectos del comportamiento, incluido el comportamiento del consumidor" (véase también Muniz \& O'Guinn, 2001).

La opinión libre de individuos en Internet permite al investigador acceder a datos provenientes de miles de individuos actuando libre y espontáneamente, lo que supone un aumento exponencial respecto a técnicas de análisis como entrevistas ofocus groups.

En este caso de estudio, la investigadora ha sido miembro del grupo desde su creación y ha ido observando y registrando sin intervenir, todas las interacciones. 
Para este estudio, una vez recopilado los datos cuantitativos, proporcionados por la comunidad misma, se han seleccionado 33 días (del 13 octubre al 14 de noviembre 2018), 231 post y 8000 entre comentarios y reacciones, que han sido analizados cuantitativamente y cualitativamente.

Para extraer y analizar los datos hemos utilizado Netvizz, un programa diseñado específicamente para investigar datos de Facebook, a través de una interfaz de aplicación (Rieder, 2013); después hemos seleccionado los comentarios y los hemos analizado a través de un análisis del contenido cualitativo, inspirado a la metodología propuesta por Toret et al. (2013), que permite tener en cuenta las emociones.

Finalmente, la entrevista en profundidad con Guido Prussia, creador de la comunidad, ha permitido matizar cualitativamente diversos aspectos.

\section{El caso de estudio: Ho sempre voglia di partire}

Ho sempre voglia di partire es una página Facebook creada en 2016 por Guido Prussia, periodista y documentalista de viajes, con una larga trayectoria profesional que se encarga de los contenidos, y el joven Alessandro Paradossi, bloguero, que maneja la parte técnica.

Guido Prussia es un personaje conocido a nivel italiano siendo el creador y presentador de diversos programas para las televisiones RAI, MEDIASET y SKY y habiendo escrito varios libros de viaje (y no) para Mondadori y Sperling.

En particular el periodista se convirtió en una verdadera estrella de la televisión a mitad de los años 90, con la emisión, en los canales Mediaset, de Hotel California, programa del cual fue autor y presentador.

El programa, una serie documental on the road en la famosa Route 66 de EEUU, presentaba el periodista viajando en moto y acompañado por diversas mujeres de aspecto agradables, generalmente modelos, visitando lugares insólitos, como el mismo Hotel California, que dio el nombre a la serie o el Área 51, o mansiones de estrellas de Hollywood.

El hecho de ser conocido podría haber influido en el éxito de la página, por eso en la entrevista en profundidad se preguntó al periodista cuánto su fama pudo influir en el éxito de la página y la respuesta fue negativa, ya que su nombre apenas aparece. Efectivamente el nombre casi no aparece, aunque en realidad Guido aparece en diferentes videos y el hecho de ser perio- 
dista de viaje y de tener una base de datos video indudablemente ayuda a la creación de contenido.

La página, en realidad, se creó por primera vez el 26 de Diciembre de 2013 con el nombre Viaggiando si impara (Viajando se aprende), cambiado inmediatamente después por Viaggiare per crescere (Viajar para crecer) y tuvo escasos éxitos, hasta su lanzamiento oficial en 2016 bajo el nombre Amo viaggiare.

Según su creador, el nombre Amo viaggiare era demasiado genérico, por eso lo cambió a Ho sempre voglia di partire.

El significado literal de este nombre sería "Siempre tengo ganas de salir", pero el verbo partire en italiano se acerca bastante más al español "despegar", por esto la traducción más adecuada nos parece "Tengo siempre ganas de viajar", pero la frase es menos genérica, sugiriendo, de alguna forma una suerte de urgencia de despegue, de viajar lejos.

Este mensaje se ve claramente representado en su pantalla inicial, donde se puede leer:

Nosotros somos los que amamos viajar, que sabemos que solo conociendo diferentes mundos, diferentes culturas, diferentes personas podemos alimentar el hambre de conciencia y conocimiento. Somos aquellos que se sienten vivos cuando se mueven. Somos aquellos para quienes el mundo representa una posibilidad infinita de ser sorprendido. Nosotros somos los que siempre queremos salir/despegar/viajar.

\title{
Figura 1 \\ Encabezado de la página
}

\author{
Ho sempre voglia di partire \\ 당 \\ Siamo quelli che amano viaggiare. \\ Che sanno che solo conoscendo mondi diversi, culture diverse, persone diverse si puo' nutrire \\ la fame di consapevolezza e conoscenza. \\ Siamo quelli che quando sono in movimento si sentono vivi. \\ Siamo quelli per cui il mondo rappresenta un infinita possibilità di sorprendersi. \\ Siamo quelli che hanno sempre voglia di partire.
}

Fuente: https://goo.gl/hTGLiJ 
En septiembre 2016, justo después de las vacaciones, empezó la publicidad en Facebook de su página con el nuevo nombre y la gente se interesó porque, según lo que afirma en la entrevista, justamente, tenían ganas de "salir" otra vez, de volver a viajar.

Actualmente la página tiene 6257209 personas alcanzadas, 3430000 visualizaciones semanales 650000 interacciones semanales.

La página, de hecho, es un magazine de viaje que - desde su principiofue concebida como un "espacio libre, donde poder hablar libremente de viajes sin constricciones mediáticas y comerciales" (entrevista a Guido Prussia, 2018). El periodista, ideó este proyecto para poder hacer lo que le gusta, es decir hablar de viajes, sin tener que responder a lógicas editoriales que, en sus propias palabras, "muchas veces matan la creatividad" o comerciales.

La página propone siete post por día: un video relacionado con el viaje pero no necesariamente sobre un destino, tres fotografías o memes, normalmente, pero no necesariamente relacionados con el mundo de los viajes, y tres artículos sobre uno o más destinos.

Los videos pueden ser o videos de viajes, a menudo piezas periodísticas del creador, Guido Prussia, o video-memes. Las fotos frecuentemente son aforismas hechos ad hoc para ser compartidos. En la Figura 2 un ejemplo que dice "Cuando estás triste los viajes deberían proporcionártelos la Seguridad Social".

\section{Figura 2}

\section{Ejemplo de fotografía}

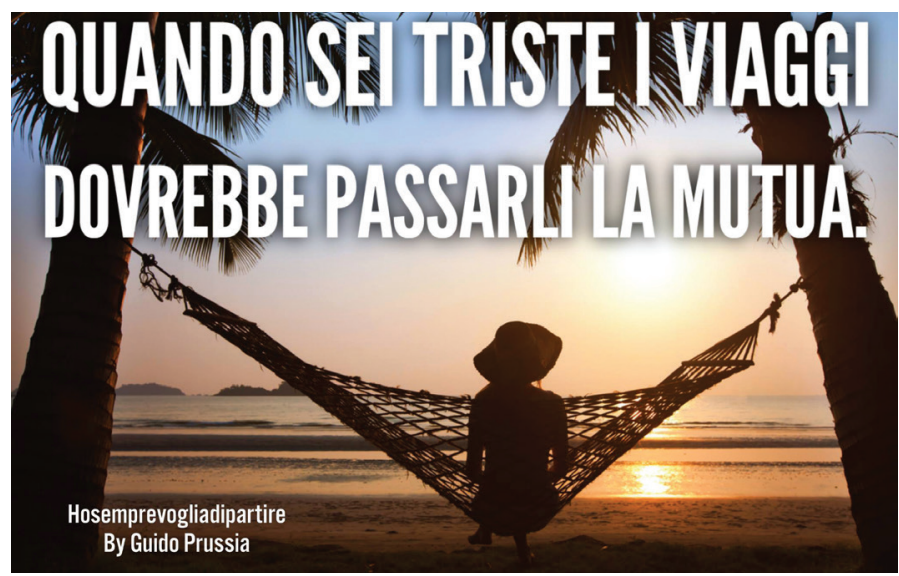

Fuente: https://goo.gl/815iu5 
Solo los artículos son siempre de estilo periodístico. Normalmente se presenta un destino, añadiendo sugerencias sobre qué ver, qué tipo de experiencias hacer, etc.

En la Figura 3 vemos un ejemplo "La casa de Heidi existe de verdad", donde el periodista sugiere de visitar los Alpes donde se encuentra una choza construida igual a la casita de Heidi, un popular dibujo animado de los años 80 .

\section{Figura 3 \\ Ejemplo de artículo}

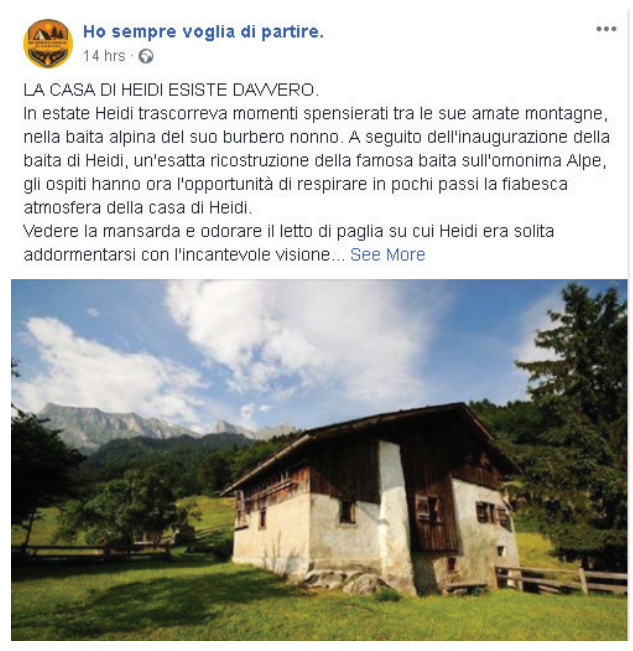

Fuente: https://goo.gl/7iGHpj

\section{Resultados}

Después de haber desglosado los datos estructurales (número de seguidores, evolución, género y edades) pasaremos al análisis de los 33 días seleccionados.

La Tabla 1 muestra la evolución en el número de seguidores.

Los datos parecen confirmar las declaraciones de Guido Prussia en la entrevista: los seguidores aumentan exponencialmente a partir de 2016, es decir a partir del cambio de nombre. 


\section{Tabla 1}

\section{Evolución de los seguidores}

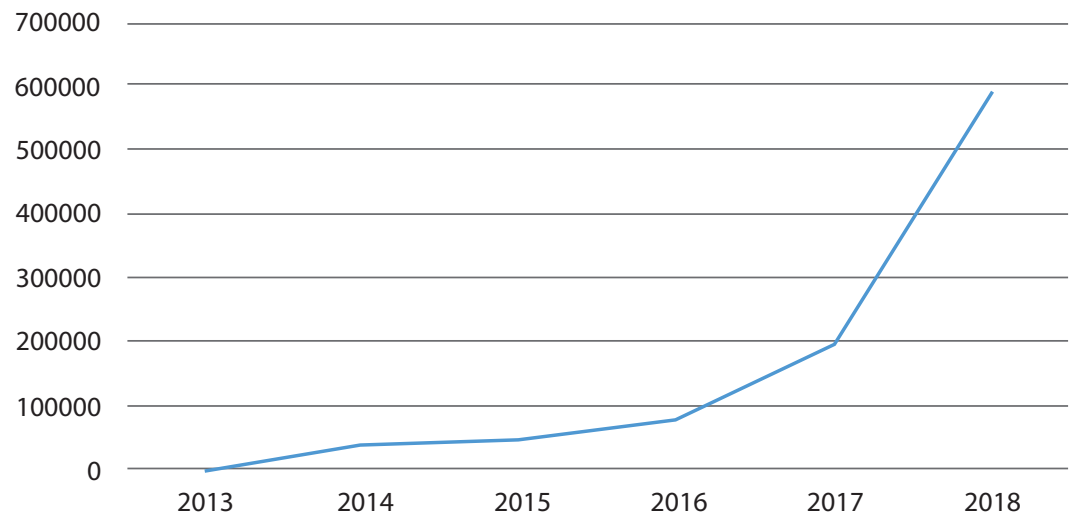

Fuente: Elaboración propia a partir de datos proporcionados por Ho sempre voglia di partire.

La Tabla 2 muestra el género de los seguidores, que es preponderantemente femenino. Las investigaciones confirman que las mujeres tienden a estar más dispuestas a compartir en red y la entrevista con el creador de la página nos confirma que desde el principio el target fue claramente femenino.

Tabla 2

Género de los seguidores

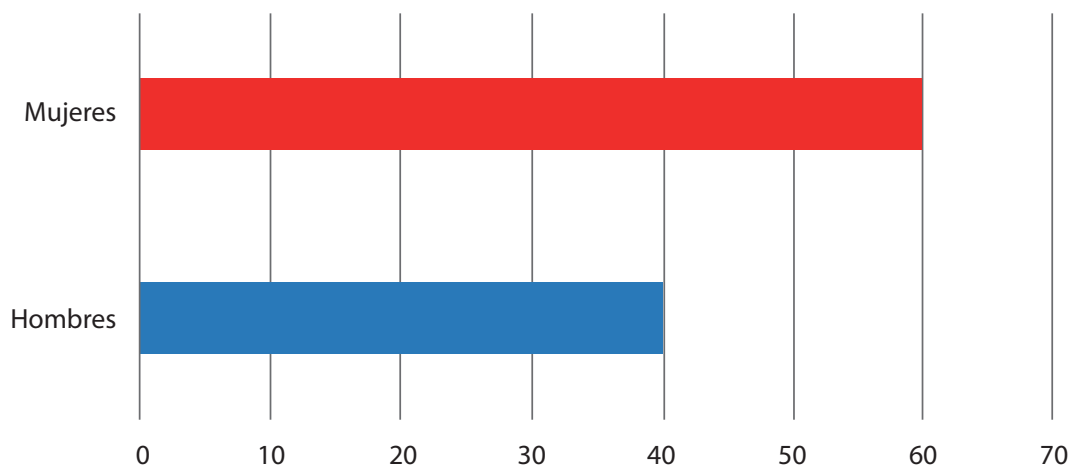

Fuente: Elaboración propia a partir de datos proporcionados por Ho sempre voglia di partire. 
Cuanto a las edades, la Tabla 3 nos muestra que, a pesar de tener seguidores de todas las edades, la mayor parte de ellos tienen entre 25 y 44 años, es decir los que se definen adultos y jóvenes adultos.

Por un lado la elección misma de la plataforma marca un target. Y Facebook se está cada vez más definiéndose como una plataforma para adultos: de acuerdo al último reporte eMarketer (2018), tan solo en Estados Unidos, Facebook perderá 2 millones de usuarios de menos de 25 años durante el 2018 ya que, sintiéndose expulsados por la presencia de los adultos (padres, tíos, abuelos, profesores) y, de alguna forma, acosados por las solicitudes de amistad transgeneracionales, los más jóvenes están migrando a Snapchat e Instagram.

Guido Prussia en la entrevista confirma, efectivamente, sentirse más cómodo, creando contenido por Facebook (es decir texto, video e fotos) que no para Instagram, y asume el riesgo de perder los más jóvenes.

\section{Tabla 3}

\section{Edades de los seguidores}

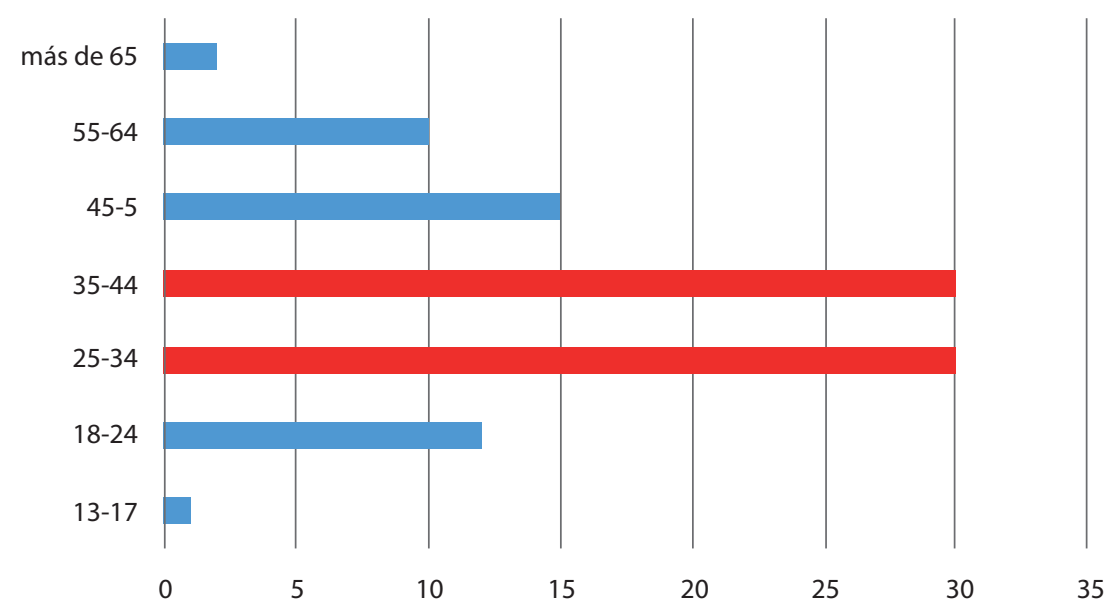

Fuente: Elaboración propia a partir de datos proporcionados por Ho sempre voglia di partire.

La Tabla 4 muestra la evolución y el número de interacciones en los 33 días de nuestro análisis. 
Tal y como se puede apreciar likes y "reacciones" van juntos y constituyen el número más grande, seguidos por los share, los números de veces que un contenido se ha compartido.

Los comentarios, en cambio, si bien en número menor, parecen seguir otra dinámica.

\section{Tabla 4}

Número de interacciones del 13 octubre

al 14 de noviembre 2018

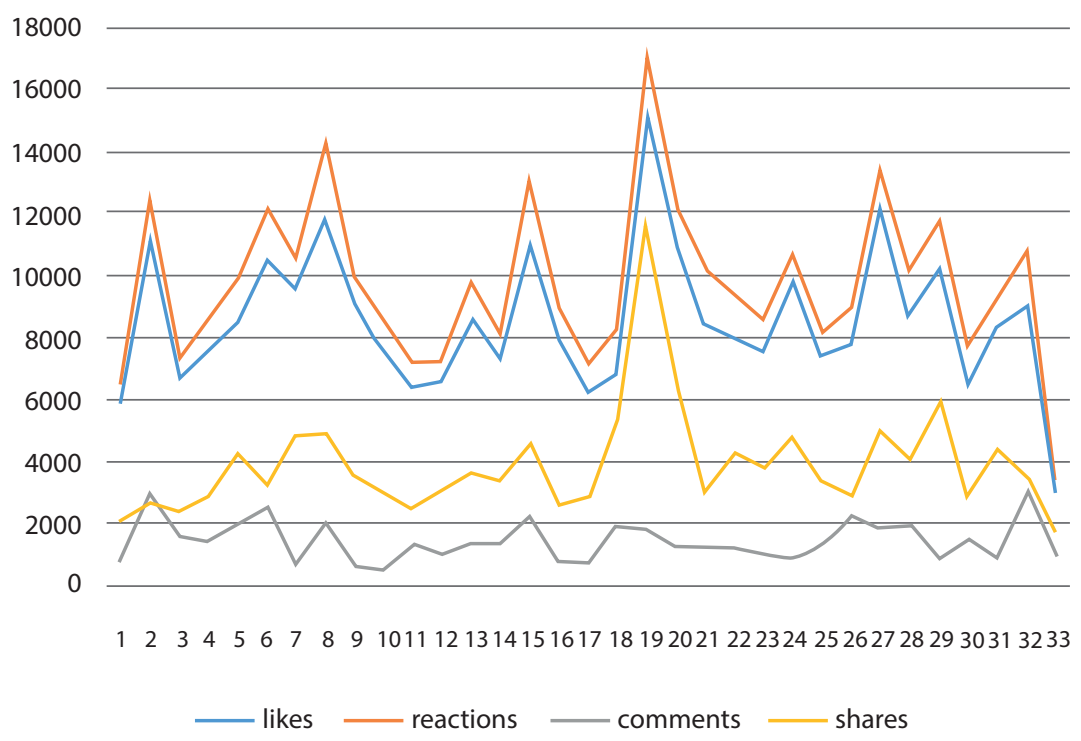

Fuente: Elaboración propia

La Tabla 5 desglosa el tipo de emoción expresada en las reacciones. Tal y como podemos apreciar la gran mayoría de reacciones pertenecen al emoticono llamado "ahahah", representado por una cara que ríe, seguido por el emoticono "love", amor, "Wow" y solo en una mínima parte la cara triste.

Si bien resulta difícil interpretar los significado detrás de las emociones (por ejemplo: ¿ la tristeza está referida al mensaje o a la nostalgia de un sitio?), resulta evidente que las emociones positivas ganan. 


\section{Tabla 5}

Recuento de las emociones expresadas en las reacciones

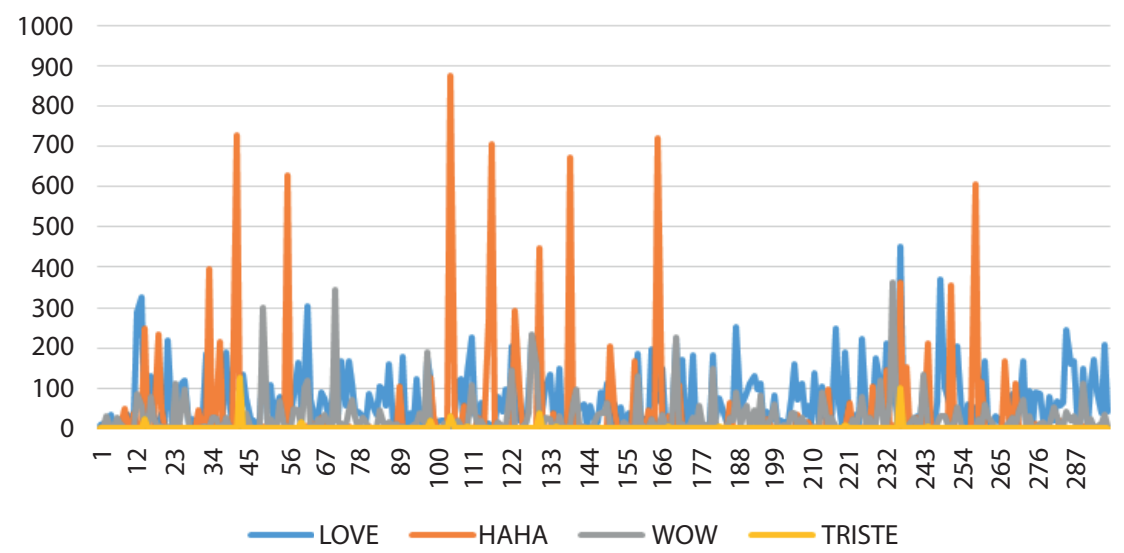

Fuente: Elaboración propia

El contenido más compartido, con más "likes" y con más reacciones ha sido el video-meme de un perrito bailando acompañado de la frase "Aquella irrefrenable felicidad que sientes cuando reservas un nuevo viaje", reportado en la Figura 5.

\section{Figura 5}

Video con más "likes" y "shares"

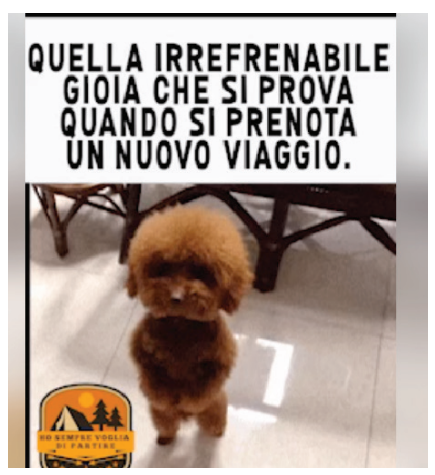

Fuente: https://goo.gl/xL4vAD 
Curiosamente este video, si bien represente una emoción referida al viajar no tiene nada que ver con el mundo del viaje estrictu sensu.

En cambio el contenido más comentado ha sido el representado en Figura 6, en el cual se pregunta a los usuarios de elegir una poción "mágica" que los llevaría a diferentes viajes.

\section{Figura 6}

Post más comentado

\section{Quale pozione vorresti bere?}

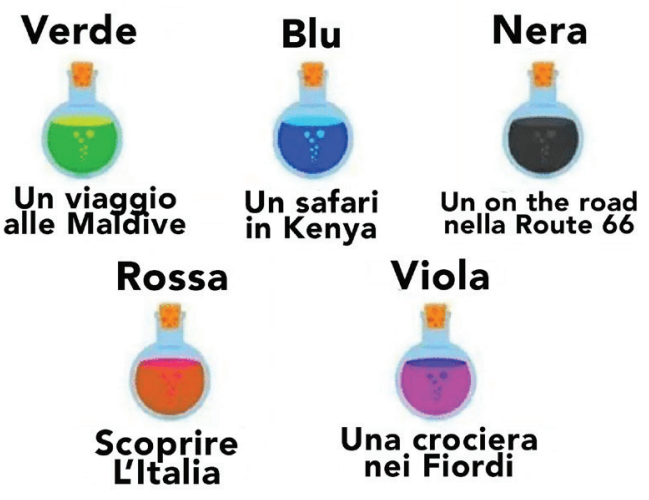

Fuente: https://goo.gl/9eMTVC

Si bien ambos contenidos hablen de viaje, curiosamente ninguno de los dos contenidos da sugerencias, especifica ni aporta información sobre ningún destino en concreto.

La Tabla 5 muestra el análisis cuantitativos de todos los comentarios colectados durante los 33 días.

Casi la mitad son tags sin comentarios, es decir un usuario simplemente taguea a otro sin más especificar.

Podemos suponer que el tag pueda significar una intención de compartir el viaje a un destino con aquella persona o la voluntad de compartir una memoria compartida, todavía en la ausencia de más información es imposible establecerlo con certidumbre. 


\section{Tabla 6 \\ Tipos de comentarios}

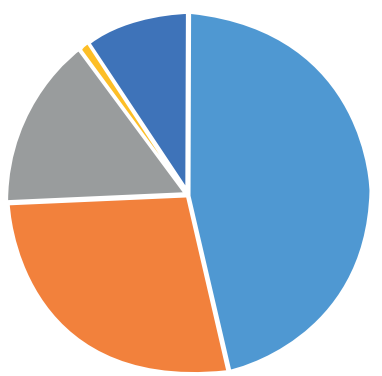

- Tag sin comentario

Sugerencias
Tag con comentario Comentario y emoticones
Emoticones

Fuente: Elaboración propia

En segundo lugar encontramos los tags con comentarios que efectivamente confirman las hipótesis anteriormente mencionada.

En la Figura 7 podemos apreciar algunos ejemplos de comentarios: el usuario ponga un tag a otro usuario para decir quiero ir aquí contigo, o para recordar un viaje pasado.

Otros usuarios comentan y añaden emojis. En la casi totalidad de los casos se trata de comentarios positivos con emojis de emociones positivas.

\section{Figura 7}

\section{Ejemplo de comentarios}

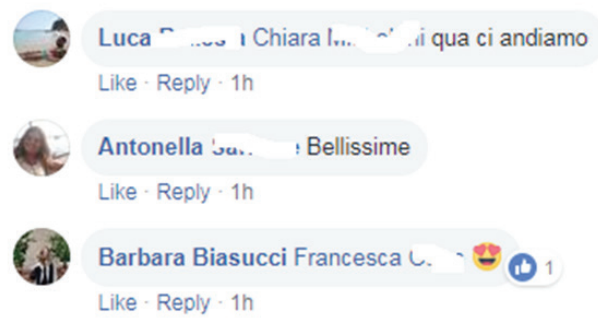

Fuente: https://goo.gl/ShqQ1b 
El análisis de contenido de los comentarios nos permite distinguir el tipo de emoción expresada.

Como podemos apreciar en la Tabla correspondiente, curiosamente, una vez más, el comentario más frecuente es "he estado ahî" en todas sus formas, sobre todo expresada con un check (el emoticon de la v verde) o con un "fatto", hecho, parecido al inglés "done".

Figura 8

Ejemplo de comentario

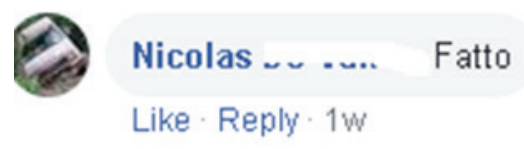

Fuente: https://goo.gl/DVBoHQ

Tabla 7

Análisis cualitativos comentarios

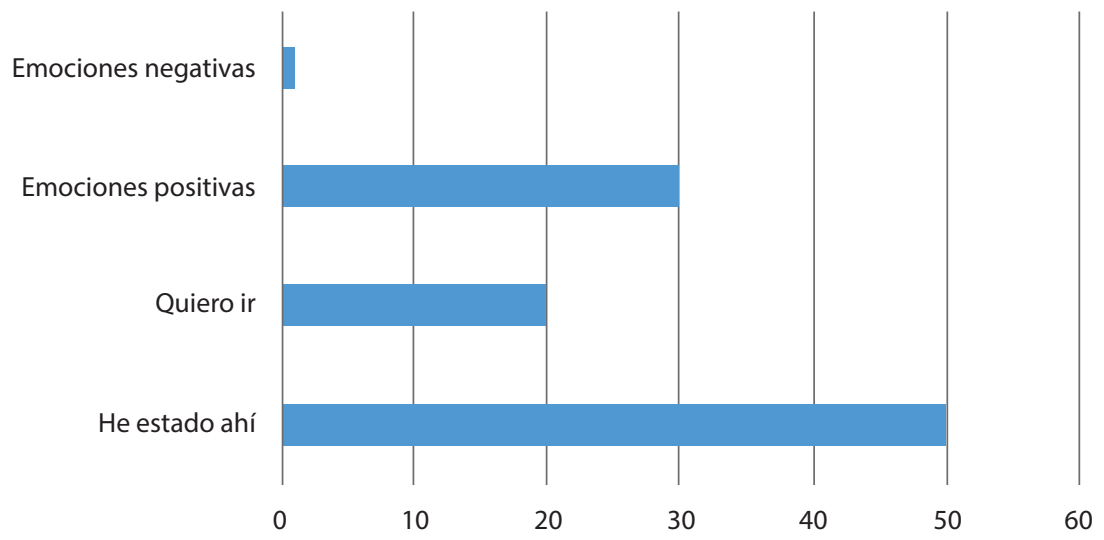

Fuente: Elaboración propia

El segundo es "quiero ir", normalmente acompañado del tag a la persona con la cual se piensa emprender el viaje y emociones positivas respeto 
al destino, expresadas con emojis o en palabras como "espectacular" "que maravilla", etc.

Las emociones negativas con prácticamente inexistentes.

En los 33 días solo encontramos dos: una, referida a unos jardines de la ciudad de Ámsterdam simplemente dice "Estos no me gustan, prefiero otras cosas", y la otra es un emoticono que llora que podría significar muchas cosas diferentes (añoranza del sitio, llorar de conmoción, etc.).

\section{Conclusión y discusión}

Los resultados de este caso de estudio de alguna forma sorprenden ya que en una comunidad de viajes hemos encontrado pocas muestras de intercambio de informaciones funcionales (por ejemplo sugerencia sobre lugares para visitar, experiencias, hoteles o restaurantes). Al revés los miembros participan principalmente compartiendo los contenidos y sobre todo tagueando a otros usuarios, afirmando haber estado u expresando su voluntad de visitar un sitio y sus emociones.

Los resultados obtenidos, por lo tanto, están indudablemente de acuerdo con los citados hallazgos de Wang y Fesenmaier (2004b) y con los recientes descubrimientos de Xiang, Du, Ma y Fan (2017), ya que es evidente que los beneficios sociales (véase la cantidad de tags) y hedónicos (la cantidad de "he estado ahí") tienen un mayor impacto en la participación de los miembros en las actividades de la comunidad en línea que los beneficios funcionales.

En particular esta aparente necesidad de "informar" a los demás miembros de haber visitado un sitio con un simple "hecho", como si de un listado de tareas se tratara, y sin ofrecer comentarios adicionales parece subrayar este valor hedonístico, de voluntad de pertenencia, muy por encima del valor funcional de ofrecer la experiencia para el beneficio de los otros.

Hace más de tres décadas, Marshall McLuhan explicó que los 'medios eléctricos' 'fríos' e inclusivos "retribalizarían" el ser humano dividiendo la sociedad en grupos de afiliación (ver, por ejemplo, McLuhan \& Watson, 1970), y este tipo de consumo social y hedonístico parece probar su teoría, así como parece concordar con la idea que las redes sociales han expandido la perspectiva de la tecnología web transformando los usuarios en "tecnocontactores" (Kozinets, 1999), que usan la tecnología como un proveedor mediado de realización individual. 
Sin embargo si definimos los beneficios sociales como la comunicación con otros miembros, la construcción de relaciones, el intercambio de ideas y opiniones y la participación (Angehrn, 1997; Preece, 2000; Wang \& Fesenmaier, 2004a), nos perderíamos la parte más importante de estas interacciones: las emociones.

Tal y como hemos podido apreciar, en efecto, la mayor parte de los comentarios se limitan, más allá de enlazar otros usuarios, a expresar emociones, sea a través del texto, sea a través del uso de emojis.

Por esta razón podemos afirmar que la clave para la comprensión de esta comunidad son las emociones: se habla de viaje no tanto en términos informativos o funcionales, sino que se comparten imágenes, videos y al fin de al cabo sensaciones.

En particular, como subraya Peyton (2014), con la emergencia del botón "like" la noción de gustar ha experimentado un cambio semiótico, desplazándose desde la esfera íntima y emocional de los individuos hacia la esfera pública. Más que un sentimiento, ahora es una acción, ya que:

En lugar de estar vinculado a una sensación interna que reacciona tácitamente a un estímulo externo, 'gustar' se ha convertido ahora en una acción racional que connota una conexión externa entre un individuo, un elemento discursivo y una instancia social (Peyton, 2014, p. 113).

Dicho de otra forma, la necesidad de comprender el significado cultural de las comunidades en línea ha crecido de manera exponencial desde la aparición de las interfaces Web 2.0.y parece que las comunidades on line, a pesar del tema que traten, o en general las redes sociales, sean capitalizadoras de la influencia emocional, de aquel intercambio de sentimientos que ha sido llamado "cultura de transmisión" (Buss \& Strauss, 2009) o "cultura de la exposición” (Munar, 2010).

Por lo tanto, aunque este estudio reflexione solo sobre un caso específico, con características específicas, pone en evidencia la necesidad, por parte de las ciencias sociales, y especialmente del conjunto de las ciencias de la comunicación de fortalecer la investigación, más allá del análisis del consumo y de los consumidores, sobre las emociones en el contexto on line.

Si es cierto, parafraseando Scolari, que todas estas transformaciones no son meramente tecnológicas sino que afectan al mundo y a la comprensión de éste por parte del sujeto, de cara a futuras investigaciones, sería importante comparar diferentes casos y contextos, para confirmar que estos ras- 
gos son compartidos. En ese sentido, es necesario tener en cuenta las hipermediaciones, estableciendo un paradigma que "debe saber moverse en un terreno discursivamente pantanoso, consolidando una sólida red de interlocutores a partir de los cuales comenzar a construir su propio recorrido epistemológico" (Scolari, 2008, p. 144).

\section{Agradecimientos}

Se agradece el staff de Ho sempre voglia di partire en la persona de su creador, Guido Prussia, por habernos otorgado expresamente el derecho a consultar los datos de su página y a reproducir sus imágenes.

\section{Bibliografía}

Almeida García, F., Peláez-Fernández, A. M., Macías, R., \& Balbuena, A. (2016). Residents' perceptions of tourism development in Benalmádena (Spain). Tourism Management, 54, 259-274. 10.1016/j.tourman.2015.11.007.

Agag, G., \& El-Masry, A. (2016a). Understanding Consumer Intention to Participate in Online Travel Community and Effects on Consumer Intention to Purchase Travel Online and WOM: An Integration of Innovation Diffusion Theory and TAM with trust. Computers in Human Behaviour. http:// dx.doi.org/10.1016/j.chb.2016.02.038.

(2016b). Understanding the Determinants of Hotel Booking Intentions and moderating Role of Habit. International Journal of Hospitality Management 54, 52-67. http://dx.doi.org/10.1016/j.ijhm.2016.01.007

Amaro, S., \& Duarte, P. (2013). Online Travel Purchasing: A Literature Review. Journal of Travel \& Tourism Marketing, 30(8), 755-782. http://dx.doi.or $\mathrm{g} / 10.1080 / 10548408.2013 .835227$

Amaro, S., Duarte, P., \& Henriques, C. (2016). Travelers' use of social media: A clustering approach. Annals of Tourism Research, 59, 1-15. https://doi. org/10.1016/j.annals.2016.03.007

Andereck, K. L., \& Caldwell, L. L. (1993). The inflaence of tourist's characteristics on ratings of infonnation sources for an attraction. En M. Uysal \& D. R. Fesenmaier (Eds.), Communication and Channel Systems in Tourism Marketing (pp. 171-190). London: The Haworth Press. 
Angehrn, A. (1997). Designing mature internet business strategies: The ICDT model. European Management Journal, 15(4), 361-369. https://doi. org/10.1016/S0263-2373(97)00016-9

Armstrong, A., \& Hagel, J. (1996). The Real Value ofOn-line Communities. Harvard Business Review, 74(3), 134-141, May-June. https://doi.org/10.1016/ B978-0-7506-7293-1.50009-3

Ayeh, J. K. (2015). Travellers' Acceptance of Consumer-Generated Media: An Integrated Model of Technology Acceptance and Source Credibility Theories. Computers in Human Behavior, 48, 173-80. https://doi.org/117.724915

Ayeh, J. K., Au, N., \& Law, R. (2013). Predicting the Intention to use Consumer Generated Media for Travel Planning. Tourism Management, 35, 132143. https://doi.org/95.05557

Baym, N. (2010). Personal Connections in the Digital Age. Malden, MA: Polity Press.

Beith,M.(2004).Living like locals.Newsweek(19-04-2004) https://bit.ly/2S4Q9YM

Bilgihan, A., Barreda, A, Okumus, F., \& Nusair, K. (2016). Consumer perception of knowledge- sharing in travel-related Online Social Networks. Tour. Manag., (52), 287-296, https://doi.org/ 62.478733

Bray, J., Schetzina, C., \& Steinbrink, S. (2006). Six travel tech trends for 2006. En Christou, E., Sigala, M. (Ed.), Social Media in Travel, Tourism and Hospitality. London: Routledge, https://doi.org/10.4324/9781315609515

Bronner, F., \& de Hoog, R. (2008). Agreement and Disagreement in Family Decision-making. Tourism Management, (29), 967. https://doi.org/10.1016/j. tourman.2007.12

Bruns, A. (2008). Blogs, Wikipedia, Second Life, and Beyond: From Production to Produsage. New York: Peter Lang.

Bryce, D., Curran, R., O’Gorman, K., \& Taheri, B. (2015). Visitors' Engagement and Authenticity: Japanese Heritage Consumption. Tourism Management, (46), 571-581. http://dx.doi.org/10.1016/j.tourman.2014.08.012

Chang, H. H., Fu, C.S., \& Jain, H.T. (2016). Modifying UTAUT and innovation diffusion theory to reveal online shopping behavior: Familiarity and perceived risk as mediators. Information Development, 32(5), 17571773. https://doi.org/10.1177/0266666915623317

Chen, G., Yang, S., \& Tang, S. (2013). Sense of virtual community and knowledge contribution in a

P3 virtual community: Motivation and experience. Internet Res., 23(1), 4-26. https://doi.org/10.13140/2.1.4684.0960 
Chung, N., \& Koo, C. (2015). The Use of Social Media in Travel Information Search. Telemat. Informatics, 32. https://doi.org/10.1016/j.tele.2014.08.005

Chung, N., Lee, H., Lee, S. J., \& Koo, C. (2015). The Influence of Tourism Website on Tourists' Behavior to Determine Destination Selection: A Case Study of Creative Economy in Korea. Technological Forecasting and Social Change, 96, 130-143. https://doi.org/10.1016/j.techfore.2015.03.004

Creswell, J. W. (2014). Research Design: Qualitative, Quantitative and Mixed Methods Approaches (4th ed.). London: Sage Publications Ltd.

Dedeke, A. (2016). Travel Web-site Design: Information Task-fit, Service Quality and Purchase Intention. Tourism Management, 54, 541-554. https://doi. org/0.1016/j.tourman.2016.01.001

Enoch, Y., \& Grossman, R. (2010). Blogs of Israeli and Danish Backpackers to India. Annals of Tourism Research, 7(2), 520-36. https://doi.org/10.1016/j. annals.2009.11.004

Ert, E., Fleischer, A., \& Magen, N. (2016). Trust and Reputation in the Sharing Economy: The Role of Personal Photos in Airbnb. Tourism Management, 55, 62-73. https://doi.org/10.2139/ssrn.2624181

Filieri, R., \& McLeay, F. (2014). E-WOM and Accommodation: An Analysis of the Factors That Influence Travelers' Adoption of Information from Online Reviews. J. Travel Res., 53 (1), 44-57. https://doi. org/10.1177/0047287513481274

Fotis, J., Buhalis, D., \& Rossides, N. (2012). Social media use and impact during the holiday travel planning process. Information and Communication Technologies in Tourism, 13-24. https://doi.org/10.1007/978-3-7091-11420_2

Gitelson, R. J., \& Crompton, J. (1983). The planning horizons and sources of infonnation used by pleasure vacationers. Journal of Travel Research, 21(3), 2-7. https://doi.org/10.1177/004728758302100301

Gretzel, U., Fesenmaier, D. R., \& O’Leary, J. T. (2006). The transfonnation of consumer behaviour. En D. Buhalis \& C. Costa (Eds.), Tourism Business Frontiers. Amsterdam; London: Buttterworth Heinemann.

Harrigan, P., Evers, U., Miles, M., \& Daly, T. (2017). Customer Engagement with Tourism

Social Media Brands. Tourism Management, 59, 597-609. https://doi.org/10.1016/j. tourman.2016.09.015

Howard, R., Restrepo, L., \& Chang, C. (2017). Addressing individual perceptions: An application of the unified theory of acceptance and use of 
technology to building information modelling. International Journal of Project Management, 35, 107-120. http://dx.doi.org/10.1016/j.ijproman.2016.10.012

Hsu, M.H., Ju, T.L., Yen, C.H., \& Chang, C.M. (2007). Knowledge sharing behavior in virtual communities: The relationship between trust, self-efficacy, and outcome expectations. International Journal Human-Computer Studies, 65(2), 153-169. http://dx.doi.org/10.1016/j.ijhcs.2006.09.003

Jacobsen, J. K. S., \& Munar, A. M. (2012). Tourist information search and destination choice in a digital age. Tourism Management Perspectives, 1, 39-47 https://doi.org/10.1016/j.tmp.2011.12.005

Kamboj, S., \& Rahman, Z. (2017). Measuring customer social participation in online travel communities: Scale development and validation. Journal of Hospitality and Tourism Technology, 8(3) 432-464. https://doi. org/10.1108/JHTT-08-2016-0041

Kang, M., \& Schuett M. (2013). Determinants of Sharing Travel Experiences in Social Media. J. Travel Tour. Mark., 30(1), 107-127. https://doi.org/10.1 080/10548408.2013.751237

Kaplan, A., \& Haenlein, M. (2010). Users of the world, unite! The challenges and opportunities of social media. Business Horizons, 53(1), 59-68. https:// doi.org/10.1016/j.bushor.2009.09.003

Kavoura, A., \& Stavrianea, A. (2015). Following and Belonging to an Online Travel Community in Social Media, its Shared Characteristics and Gender Differences. Procedia - Soc. Behav. Sci., 175, 515-521. https://doi. org/10.1016/j.sbspro.2015.01.1231

Kavoura, A., \& Borges, M. T. T. (2016). Understanding online communities on social networks via the notion of imagined communities: the case of Tripdvisor. International Journal of Web Based Communities, 12(3), 238-261. https://doi.org/10.1504/IJWBC.2016.077759

Kim, W. G., Lee, C., \& Hiemstra, S. J. (2004). Effects of an online virtual community on customer loyalty and travel product purchases. Tourism management, 25(3), 343-355. https://doi.org/10.1016/S0261-5177(03)00142-0

Kozinets, R. V. (1999). E-tribalized marketing?: the strategic implications of virtual communities of consumption. European Management Journal, 17(3), 252-264, https://doi.org/10.1016/S0263-2373(99)00004-3

Li, C., \& Bernoff, J. (2008). Groundswell. Winning in a world transformed by social technologies. 1st ed., Boston, MA, Harvard Business Press. 
Litvin, S. W., \& Dowling, K. M. (2017). Tripadvisor and hotel consumer brand loyalty. Current Issues in Tourism, 1-5. 10. https://doi. org/1080/13683500.2016.1265488

Litvin, S. W., Goldsmith, R. E., \& Pan, B. (2008). Electronic word-of-mouth in hospitality and tourism management. Tourism Management, 29(3), 458-468. https://doi.org/10.1016/0160-7383(92)90127-B

McLuhan, M., \& Watson, W. (1970). From Cliché to Archetype with Wilfred Watson. New York: Pocket Book.

Mayo, E. J., \& Jarvis, L. P. (1981). The Psychology of Leisure Travel. Boston: CBI Publishing.

Mariampolski, H. (2005). Ethnography for marketers: A guide to consumer immersion. London: Sage.

Mansfeld, Y. (1992). From motivation to actual travel. Annals of Tourism Research, 19, 399-419. https://doi.org/10.1016/0160-7383(92)90127-B

Mill, R. C., \& Morrison, A. M. (2002). The tourism system (4th ed.). Dubuque, Iowa: Kendall/Hunt Pub.

Mohd-Any, A. A., Winklhofer, H., \& Ennew, C. (2015). Measuring Users' Value Experience on a Travel Website (e-value): What Value is Cocreated by the User? Journal of Travel Research, 54(4), 496-51. https://doi. org/10.1177/0047287514522879

Munar, Ana Maria (2010). Digital Exhibitionism: The Age of Exposure. Culture Unbound, 2, 401- https://doi.org/22. 10.3384/cu.2000.1525.10223401

Muniz, A. M., Jr., \& O’Guinn T. C. (2001). Brand Community. Journal of Consumer Research, 27(4), 412-432. https://doi.org/10.1086/319618

Olsen, M. D., \& Connolly, D. J. (2000). Experience-based Travel. Cornell Hotel and Restaurant Administration Quarterly, 41(1 ), 30-40. https://doi. org/10.1177/001088040004100121

Pantelidis, L. S. (2010). Electronic meal experience: A content analysis of online restaurant comments. Cornell Hospitality Quarterly, 51(4), 483-91. https://doi.org/10.1177/1938965510378574

Peyton, T. (2014). Emotion to action?: Deconstructing the ontological politics of the "Like" button. En T. Benski \& E Fisher (Eds.), Internet and Emotions (pp. 113-129). New York: Routledge.

Powers, M. J. (1997). How to program a Virtual Community. Emeryville, Calif.: Ziff-Davis Press.

Preece, J. (2000). Online communities: designing usability, supporting sociability. New York, John Wiley. 
Prebensen, N. K., Kim, H., \& Uysal, M. S. (2016). Co-creation as Moderator Between the Experience Value and Satisfaction Relationship. Journal of Travel Research, 5(7), 934-945. https://doi.org/10.1177/0047287515583359

Pudliner, B. A. (2007). Alternative literature and tourist experience: travel and tourist Weblogs. Journal of Tourism and Cultural Change, 5(1), 46-59. https://doi.org/10.2167/jtcc051.0

Qualman, E. (2009). Socialnomics: how social media transforms the way we live and do business. New Jersey: John Wiley \& Sons.

Rashidi, T.H., Abbasi, A., Maghrebi, M., Hasan, S., \& Waller, T.S. (2017). Exploring the capacity of social media data for modelling travel behaviour: Opportunities and challenges. Transportation Research Part C: Emerging Technologies, 75, 197-211. https://doi.org/10.1016/j.trc.2016.12.008 -

Reypens, C., Lievens, A., \& Blazevic, V. (2016). Leveraging Value in Multi-stakeholder Innovation Networks: A Process Framework for Value Co-creation and Capture. Industrial Marketing Management, 56, 40-50. https:// doi.org/10.1016/j.indmarman.2016.03.005

Rieder, B. (2013). Studying Facebook via data extraction. En Proceedings of the 5 th Annual ACM Web science conference. WebSci'13. Paris, May. 2-4, pp. 346-355. https://doi.org/10.1145/2464464.2464475

Rheingold, H. (1991). Virtual reality. New York: Summit Books.

Roque, V. \& Raposo, R. (2015). Social media as a communication and marketing tool in tourism: an analysis of online activities from international key player DMO. Anatolia. https://doi.org/10.1080/13032917.2015.108320 9 .

Rosenblatt, B. (1997). Virtual Communities: The benchmark for success online? Recuperado de https://bit.ly/2GuKSIE (18-05-2006).

Shneiderman, B. (2015). Building Trusted Social Media Communities: A Research Roadmap for Promoting Credible Content. En Roles, Trust, and Reputation in Social Media Knowledge Markets. Springer Cham Heidelberg, New York, Dordrecht, London: Springer International Publishing.

Scolari, C. (2008). Hipermediaciones. Elementos para una teoría de la comunicación digital interactiva. Barcelona: GDISA.

Shelton, K., \& McNeeley, T. (1997). Virtual communities companion. Albany: Coriolis Group Books.

Sirakaya, E., \& Woodside, A. G. (2005). Building and testing theories of decision making by travellers. Tourism Management, 26(6), 815-832. https://doi. org/10.1016/j.tourman.2004.05.004 
Smith, M. A., \& Kollock, P. (1999). Communities in cyberspace. London; New York: Routledge.

Sparks, B. A., \& Browning, V. (2011). The impact of online reviews on hotel booking intentions and perception of trust. Tourism Management, 32(6), 1310-1323. https://doi.org/10.1016/j.tourman.2010.12.011

Sotiriadis, M. (2017). Sharing tourism experiences in social media: A literature review and a set of suggested business strategies. International Journal of Contemporary Hospitality Management, 29(1), 179-225, https://doi. org/10.1108/IJCHM-05-2016-0300

Toret, J. et al. (2013). Tecnopolítica: la potencia de las multitudes conectadas. Catalunya: IN3 Working Paper Series. Recuperado de https://goo.gl/VqGK3t

Tussyadiah, I. P., \& Fesenmaier, D. R. (2008). Marketing places through first-person stories. An analysis of Pennsylvania roadtripper blog. Journal of Travel \& Tourism Marketing 25, https://doi.org/10.1080/10548400802508358

Urry, J. (1990). The Tourist Gaze. London: Sage.

ogt, C., \& Fesenmaier, D. R. (1998). Expanding the functional information search model. Annals of Tourism Research, 25(3), 551-578. https://doi. org/10.1016/S0160-7383(98)00010-3

Volo, S. (2010). Bloggers' reported tourist experiences: Their utility as a tourism data source and their effect on prospective tourists. Journal of Vacation Marketing, 16(4), 297-311. https://doi.org/10.1177/1356766710380884

Wang, Y., Yu, Q., \& Fesenmaier, D. R. (2002). Defining the virtual tourist community: implications for tourism marketing. Tourism Management, 23(4), 407-417. https://doi.org/10.1016/S0261-5177(01)00093-0

Wang, Y., \& Fesenmaier, D. R. (2004a). Modeling participation in an online travel community. Journal o/Travel Research, 42(3), 261-270. https://doi. org/10.1177/0047287503258824

(2004b). Towards understanding members' general participation in and active contribution to an online travel community. Tourism Management, 25(6), 709-722. https://doi.org/10.1016/j.tourman.2003.09.011

Wang, D., Fesenmaier, D. R., Werthner, H., \& Wöber, K. (2010). The journal of information technology \& tourism: A content analysis of the past 10 years. Information Technology \& Tourism, 12, 3-16: https://doi.org/10.3727/1 09830510X12747489979547

Werthner, H., \& Ricci, F. (2004). E-commerce and tourism. Commun. ACM. 47, 101-105. https://doi.org/10.1145/1035134.1035141. 
Woodside, K. (1990). An approach to studying local government autonomy: The Ontario experience. Canadian, Public Administration, 33, 198-213. https://doi.org/10.1111/j.1754-7121.1990.tb01393.x

Xiang, Z., Du, Q., Ma, Y., \& Fan, W. (2017). A comparative analysis of major online review platforms: Implications for social media analytics in hospitality and tourism. Tourism Management, 58, 51-65. https://doi.org/10.1016/j. tourman.2016.10.001

Yu, Z., Xu, H., Yang, Z., \& Guo, B. (2016). Personalized travel package with multi-point-of-interest recommendation based on crowdsourced user footprints. IEEE Transactions on Human-Machine Systems, 46(1), 151-158, https://doi.org/10.1109/THMS.2015.2446953

Fecha de recepción: 2018/11/20; Fecha de aceptación: 2019-02-18;

Fecha de publicación: 2019/03/01 